\title{
Meteor velocity determination with plasma physics
}

\author{
L. P. Dyrud ${ }^{1}$, K. Denney ${ }^{1}$, S. Close ${ }^{1,3}$, M. Oppenheim ${ }^{1}$, J. Chau ${ }^{2}$, and L. Ray ${ }^{1}$ \\ ${ }^{1}$ Center for Space Physics, Boston University, USA \\ ${ }^{2}$ Jicamarca Radio Observatory, Peru \\ ${ }^{3}$ MIT Lincoln Laboratory, MA, USA
}

Received: 23 October 2003 - Published in Atmos. Chem. Phys. Discuss.: 27 February 2004

Revised: 28 April 2004 - Accepted: 7 May 2004 - Published: 3 June 2004

\begin{abstract}
Understanding the global meteor flux at Earth requires the measurement of meteor velocities. While several radar methods exist for measuring meteor velocity, they may be biased by plasma reflection mechanisms. This paper presents a new method for deriving meteoroid velocity from the altitudinal extent of non-specular trails. This method employs our recent discoveries on meteor trail plasma instability. Dyrud et al. (2002) demonstrated that meteor trails are unstable over a limited altitude range, and that the precise altitudes of instability are dependent on the meteoroid that generated the trail. Since meteor trail instability results in field aligned irregularities (FAI) that allow for radar reflection, non-specular trail observations may be used to derive velocity. We use ALTAIR radar data of combined head echos and non-specular trails to test non-specular trail derived velocity against head echo velocities. Meteor velocities derived from non-specular trail altitudinal width match to within $5 \mathrm{~km} / \mathrm{s}$ when compared with head echo range rates from the same meteor. We apply this technique to Piura radar observations of hundreds of non-specular trails to produce histograms of occurrence of meteor velocity based solely on this non-specular trails width criterion. The results from this study show that the most probable velocity of meteors seen by the Piura radar is near $50 \mathrm{~km} / \mathrm{s}$, which is comparable with modern head echo studies.
\end{abstract}

\section{Introduction}

Recent observations show that sensitive radars, such as Jicamarca, ALTAIR, Arecibo, Millstone, MU, Sondrestrom and EISCAT are capable of detecting complex meteor phenomenon called head echos and non-specular trails (Close et al., 2000; Mathews et al., 1997; Chapin and Kudeki, 1994;

Correspondence to: L. P. Dyrud

(ldyrud@bu.edu)
Zhou et al., 2001). Much of the existing meteor research has been conducted with low power meteor radars that reflect from trails perpendicular to the radar beam (specular meteor trails)(Ceplecha et al., 1998). However, sensitive, large aperture radars are capable of detecting plasma generated from direct meteor entry (as seen in Fig. 1). These echos are characterized by a slanted line on radar RTI images, called head echos. Head echos are often followed by trail reflections, called non-specular trails, which occur, despite the fact that many trails are roughly aligned with the radar beam. Nonspecular trail echos are attributed to coherent radio scatter from plasma turbulence-generated field aligned irregularities (FAI) in electron density (shown in Fig. 1)(Zhou et al., 2001; Dyrud et al., 2002).

This paper presents the first time radar observations of non-specular meteor trails have been used for the derivation of meteoroid properties. We demonstrate the technique and results from using non-specular trails to derive the velocity of the meteoroid that produced the trail. Before presenting our technique and results, the following paragraphs review recent work by our group and others on meteor head echos and non-specular trails.

\section{$1.1 \quad$ Head echos}

The plasma formed in the vicinity of a meteoroid as it descends through the Earth's atmosphere allows for a radar reflection. These radar head echoes enable an observer to determine meteoroid positions, speeds/velocities, and decelerations with unprecedented sensitivity and precision (Close et al., 2002; Janches et al., 2000a). Over the past few years, nearly every high powered radar has conducted a study of meteor head echos, many of which were prompted by the spectacular Leonid meteor shower of 1998. These recent campaigns discovered that no significant net increase in meteor flux occurred during the peak shower time, or when pointing toward the shower radiant. These results indicate 


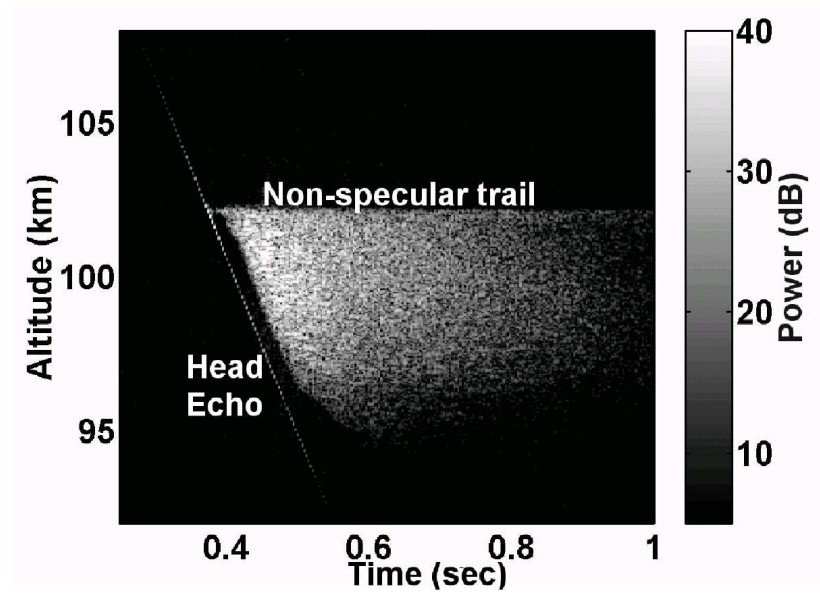

Fig. 1. Altitude-time-intensity image of a head and subsequent nonspecular echos over extended range from ALTAIR VHF Radar. Figure reproduced from Close et al. (2002).

that large aperture radar facilities easily observe the impact of the much smaller particles that comprise the sporadic meteor flux (Erickson et al., 2001; Hunt et al., 2001; Heinselman, 2001). This is not true for low power meteor radars or the naked eye, which see a large increase in meteor flux during shower periods (Brown et al., 1998). This clearly demonstrates the ability of large radars to study the dominant and poorly understood source of meteor flux.

Janches et al. (2000b) used the Arecibo UHF radar to measure some of the first micrometeor decelerations. These data combined with detailed line of sight velocity measurements yielded estimates for meteor masses between $10^{-5}-10^{2} \mu \mathrm{gm}$. Mathews et al. (2001) used these results to estimate the yearly mass flux into the upper atmosphere. These two studies provided the only measurements to date on the global micrometeor flux in this mass range, but also generated considerable controversy. These results suggest that we re-estimate the average meteor velocity upward to $\sim 50 \mathrm{~km} / \mathrm{s}$ instead of $\sim 20 \mathrm{~km} / \mathrm{s}$, and the global mass flux downward to approximately 10 tons from 100 . The estimated average meteor velocity of $\sim 20 \mathrm{~km} / \mathrm{s}$ results from specular meteor radar observations (Taylor, 1995). These high velocity meteors are detected by all the other large radar facilities (Close et al., 2000; Erickson et al., 2001; Heinselman, 2001).

How well head echo measurements sample the meteor flux is a currently debated topic. For example, Hunt et al. (2004) suggests that head echo measurements preferentially detect more energetic meteoroids over less energetic ones, and presents a method of correcting this bias when measuring the velocity distribution of meteors at Earth. Accurate estimates of average meteor mass and velocity are of crucial importance for modelers attempting to characterize the meteor effects on the ionosphere-thermosphere-mesosphere system. Finally, Close et al. (2002) has presented detailed observations of meteor head and trail echoes including the only observation of a meteor head echo at three frequencies. The research presented here will provide a complimentary method to head echos for deriving meteor velocity. Since the radar scatter from non-specular meteor trails results from different processes than head echos, any observation bias that may be of concern for head echos does not necessarily apply to non-specular trails.

\subsection{Non-specular trails}

Chapin and Kudeki (1994) were the first to present observations and interpretation of non-specular trails that suggested plasma instability as the cause for radio reflection. These observations were coincident with electrojet backscatter and were interpreted as a two-stream or Farley-Buneman instability driven by the presence of the electrojet $\boldsymbol{E}$-field. Oppenheim et al. (2000) used plasma simulations and theory to show that meteor trails do become Farley-Buneman gradient-drift (FBGD) unstable and eventually develop turbulent plasma structures. Recently, using the steerable MU radar, Zhou et al. (2001) demonstrated that non-specular trails were easily detectable when the radar pointed perpendicular to $\boldsymbol{B}$, while none were detected pointing parallel to $\boldsymbol{B}$. These results provide dramatic support for the idea that nonspecular trails are radio scatter from FAI. Dyrud et al. (2001) showed that 2-D plasma theory predicts that, at high altitudes (typically $>95 \mathrm{~km}$ ), meteor trails expand considerably more rapidly than expected due to the development of the same instability driven turbulence that generates FAI. Dyrud et al. (2002) argued that the $\sim 20 \mathrm{~ms}$ delay between head echo and non-specular trail (shown in Fig. 1) results from a turbulent trail timescale. This paper also demonstrated that only a portion of the trail is unstable and that characteristics such as meteor velocity and composition dictate the altitudes of instability. These results allow researchers to use non-specular trails to further diagnose meteor properties in completely new ways. This paper is an example of just one of these techniques. We will show that non-specular trails can be used, with reasonable accuracy, for the determination of the meteoroid speed that produced the trail.

\section{Non-specular trail altitude range}

Dyrud et al. (2002) examined the altitude range of head echo and trail pairs observed by the ALTAIR VHF radar to show that head echo scattering occurs at both higher and lower altitudes than the non-specular trails, and that all non-specular trail observations are confined within a $95-105 \mathrm{~km}$ altitude range. This same work presented a model for meteor trail formation and stability which showed that only a portion of a meteor trail is predicted unstable to the Farley-Buneman gradient drift instability. The larger purpose of the present paper is to demonstrate that we can apply the theory presented in 
Dyrud et al. (2002) that non-specular trail altitude range depends on the velocity of the meteor that generated it.

Figure 2 is a cartoon diagram demonstrating the most obvious meteor trail variation as a function of altitude; the trail radius. Meteor trails will have larger radii at higher altitudes because ablated meteor particles will random walk through the less dense atmosphere to larger distances as they cool. The details of these parameters are dependent on meteoroid properties such as meteor velocity and composition. It is this variability with altitude which causes only a portion of the plasma column to become unstable. Before we continue the main thrust of this research, we divert for a discussion on the initial radius of meteor trails which plays a critical role on the dependency of non-specular meteor trail altitude range on velocity.

\subsection{Initial radius}

As a meteor impacts the upper atmosphere, it ablates particles with substantial kinetic energy, i.e. an individual particle travelling at meteor velocities. These particles usually ionize upon their first collision and then thermalize in less than a millisecond at $100 \mathrm{~km}$ altitude (Baggaley and Webb, 1977). During the thermalization process the line density expands to an initial radius, $r_{i}$. It is during this stage that the original meteoroid velocity plays an important role. Faster meteoroids ablate particles with higher velocity that become "hotter" trail ions. These hot ions will thermalize to larger radii than particles produced from slow meteors.

The literature contains several models for calculating this so-called initial radius of meteor trails, $r_{i}$. However, a long standing disparity exists between radar derived initial radii, and those obtained theoretically. Radar derived estimates were based on knowing diffusion rates, while theoretical estimates are based on inelastic meteor ion collision calculations. Table IX of Ceplecha et al. (1998) shows that above $\sim 95 \mathrm{~km}$ the theoretical values of initial radii exceed those obtained from specular radar observations by a factor of 2 to 15.

Due to this discrepancy Oppenheim et al. (2003b) evaluated the two standard models for trail radius. The first, theoretically derived by Bronshten (1983) is

$r_{i}=\frac{2.845 \times 10^{18} v^{6}}{n_{\text {atmos. }}}$

The second model, derived from observations of specular trail diffusion rates, gives a much weaker dependence on trail radius with altitude (Baggaley, 1981),

$r_{i}=$ cn $_{\text {atmos. }}^{-.25} v^{.6}$,

where $c=.78\left(n_{0}^{-.25} v_{0}^{.6}\right)^{-1}, n_{0}$ is the atmospheric density at $90 \mathrm{~km}$, and $v_{0}$ is the meteor velocity. The results from Oppenheim et al. (2003b) showed that the Bronshten model was much better at describing head echo and non-specular trail observations. We therefore continue to rely on the Bronshten

\section{Meteor Trail}

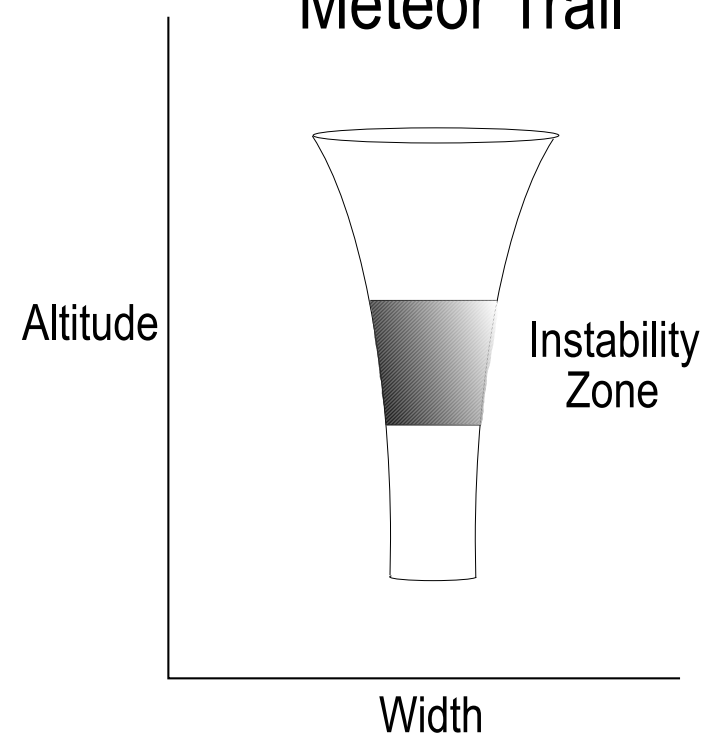

Fig. 2. Cartoon picture of the fluid expansion stage of a meteor trail, demonstrating the zone of instability. Meteor trail plasma columns expand to a greater radius at higher altitudes due to decreasing atmospheric densities with altitude. For most meteoroids this zone of instability falls between $95-105 \mathrm{~km}$.

model for our evaluations of meteor trail velocity. This is not to suggest that Bronshten had the last word on meteor trail initial radius, but that using that model gives a fairly accurate description of non-specular trails. There is however, room for improvement of initial radius models. We particularly need a model that incorporates the physics of ablated particles of different composition/mass.

\subsection{Trail instability as a function of velocity}

Figure 3 shows the predicted growth rate of the meteor trail FBGD instability as a function of altitude and meteor velocity. Meteor velocity becomes a criterion for instability due to the dependency of initial radius on meteor velocity, as shown in Eq. 1. The main idea is that faster meteors ablate particles with higher energies, which generate trails that thermalize to larger radii. These thicker trails will have weaker density gradients, which will drive instability over a shorter altitude range. First, this plot demonstrates that these weakening density gradients with altitude results in meteor trail instability over a limited altitude range between $\sim 95-105 \mathrm{~km}$. Second, this plot shows that the actual altitude range for instability depends on meteor trail velocity. There should be an observable difference in the altitude range of non-specular trails generated by $\sim 60$ and $\sim 20 \mathrm{~km} / \mathrm{s}$ meteors.

Several simplifying assumptions were made in producing Fig. 3. Most importantly this figure includes no meteor ablation or ionization physics, and simply shows the altitudes of plasma instability for a gaussian shaped plasma column with 


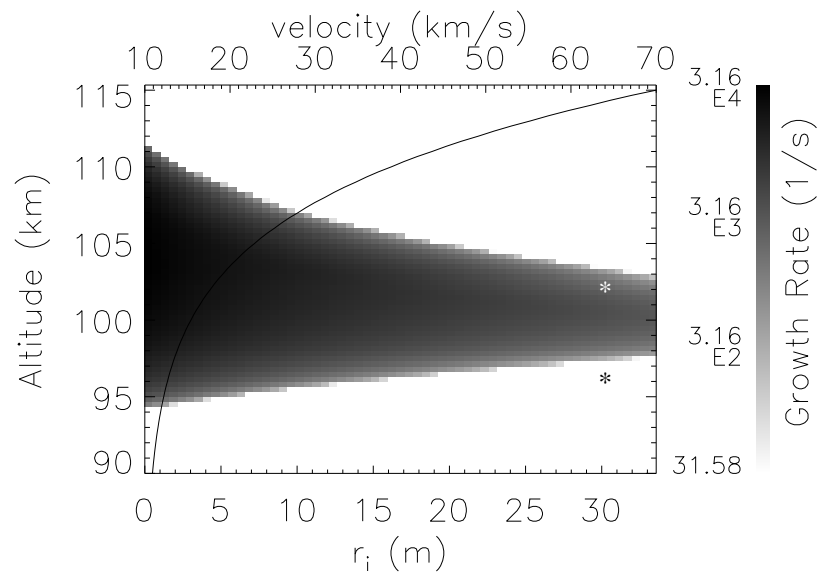

Fig. 3. Plot of instability growth rate for the FBGD instability as a function meteor velocity. The line indicates the trail initial radius at each altitude for a meteor travelling $60 \mathrm{~km} / \mathrm{s}$. Asterisks (*) mark the altitude range of the VHF trail shown in Fig. 1 which had velocity of $63 \mathrm{~km} / \mathrm{s}$.

a peak density equal to 100 times the ionospheric plasma density, and with radius equal to the the initial radius. We constructed more sophisticated models of meteor physics that include ablation, and find that there is only subtle change in the instability physics or the predicted altitude range for non-specular trails. Figure 4 shows results from this model by comparing meteor line density and expected instability. The top plot is line density as a function of altitude and meteor velocity, as produced by an ablation and ionization model. This figure was made by running the ablation equations from Lebedinets et al. (1973) for a $10 \mu \mathrm{g}$ particle and then calculating the line density (electrons per meter) using the ionization probability published by Jones (1997). The resulting line density is then expanded to the initial radius and tested for stability in the same manner as figure for 3 . The resulting growth rates are shown in the lower plot of Fig. 4 . This plot shows that the approximated plasma columns used to produce Fig. 3 capture the main physics for the prediction of non-specular trails as a function of altitude and meteor velocity, and that the predicted zone of instability does not vary by more than $1-2 \mathrm{~km}$ between the two models. One consistent change is that the lower limit of instability is more constant with velocity. This change results from limiting expected instability to wavelengths longer than twice the local debye length, which is done to approximate the kinetic solution for meteor trail FBGD instability (see Oppenheim et al., 2003a, for an explanation of this process). We point out here that Figs. 4 and 3 remain comparable for any meteor larger than about $10 \mu \mathrm{g}$, but begin to differ below this size. The altitude range of instability for smaller meteors at high velocities (larger than about $50 \mathrm{~km} / \mathrm{s}$ ) are smaller because such particles are completely ablated before exiting the lower altitude of the instability zone. In observations of head echos

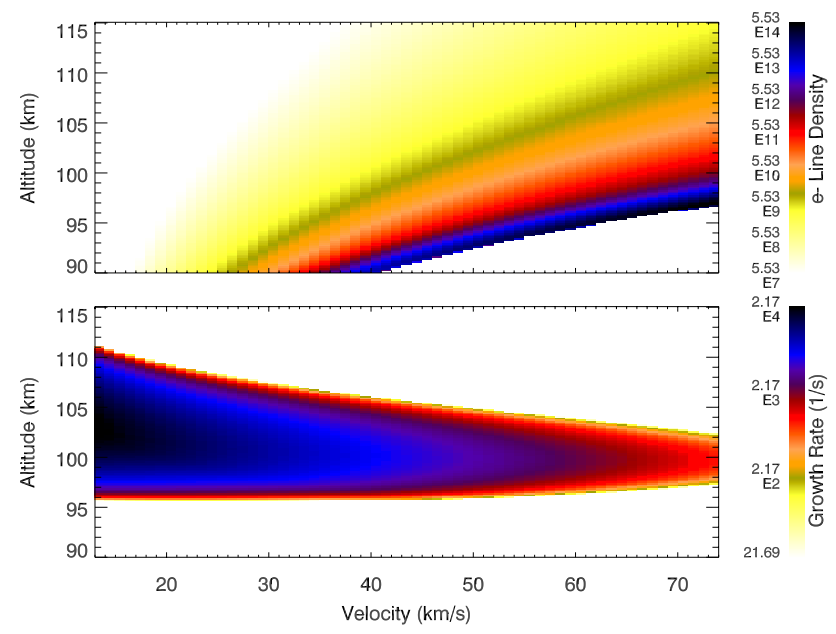

Fig. 4. The top plot shows meteor trail electron line density as a function of altitude and meteor velocity, as produced by an ablation and ionization model. The upper left portion of the plot is not devoid of density, but shows the lowest color scale. The lower plot shows the instability growth rate for the FBGD instability of a plasma column with a the line density from the top plot.

and non-specular trails, such a meteor will show the head echo and non-specular trail terminating at the same altitude.

While this paper focuses on the variation of meteor instability altitude with velocity, this altitude range is theoretically effected by additional factors. Our calculations show that the actual altitudes of meteor instability should depend on trail composition. Different ion composition shifts the altitude of trail instability up and down by a few $\mathrm{km}$, but does not effect the altitudinal extent of the trail, and therefore velocity measurements using only extent. We hope to further investigate this dependency on trail ion composition in the future. Additional meteoroid properties such as entry angle and mass do not significantly effect altitudinal extent except regarding how these parameters effect where ionization is created. The effect of this is discussed in the next paragraph.

We would like to include a caveat to the calculations for trail instability. The predictions for instability, and therefore non-specular trails described in this paper rely on a 2-D analysis of the formation of trail ambipolar electric fields in the plane perpendicular to the magnetic field. The ambipolar field together with the diamagnetic drift are responsible for driving instability. Such an analysis is truly only correct for meteor trails that are perfectly aligned with the geomagnetic field. The ambipolar electric field that develops in 3-D will be different for trails that have an arbitrary angle of inclination to $\boldsymbol{B}$. These fields must are found by solving $\nabla \cdot \boldsymbol{J}$, in 3-D which is not easily done analytically. The main issue with a 3-D solution, is that when the angle between the trail axis and the magnetic field becomes sufficiently large, electrons, with their very slow perpendicular mobility, will preserve charge neutrality by travelling long distances along 

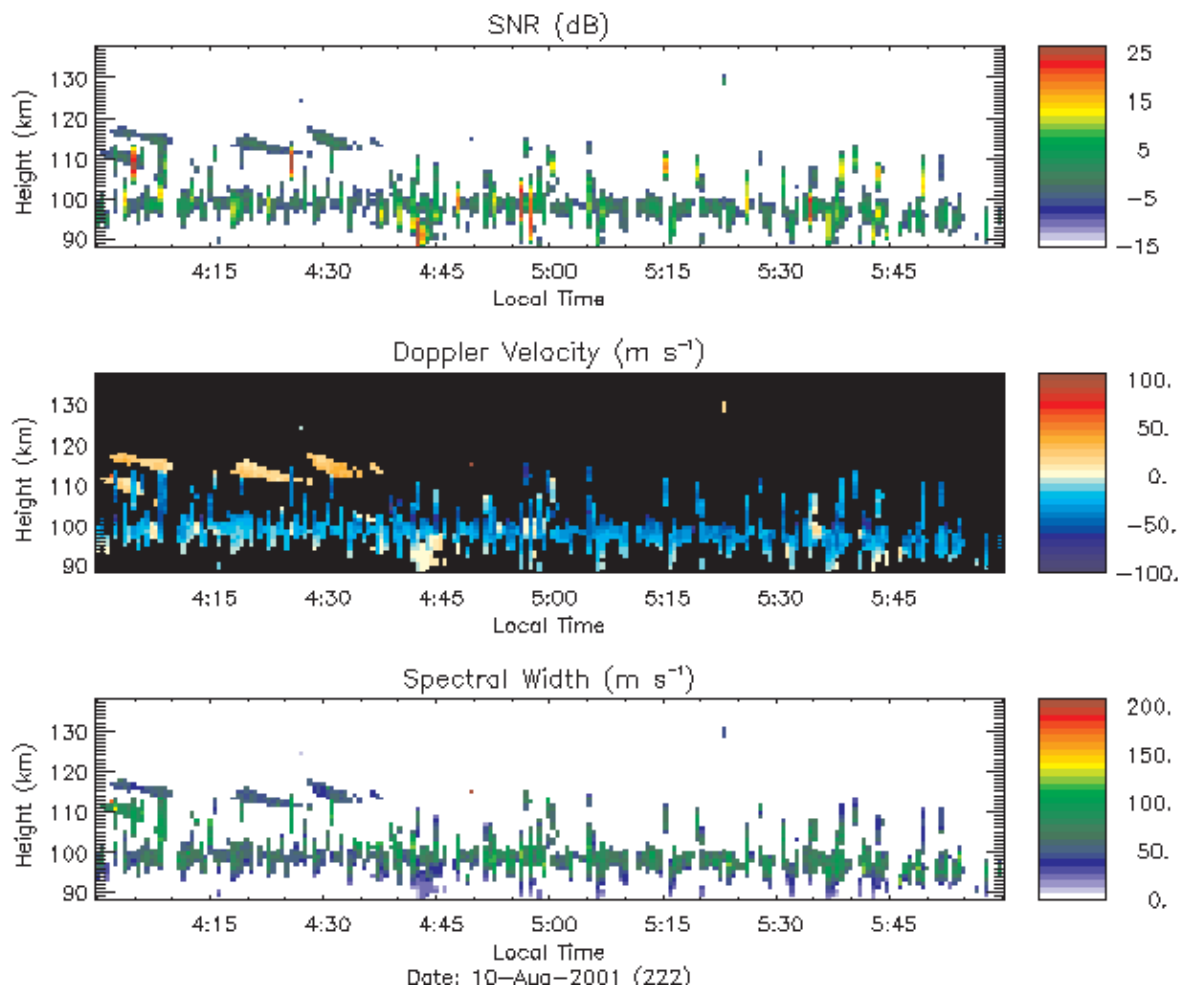

Fig. 5. $2 \mathrm{~h}$ of Radar data from 4:00-6:00 LT shows hundreds of non-specular trails. The data is averaged every minute which precludes plotting of head echos which are too brief to have significant average power. The top panel shows SNR, the middle panel is doppler velocity, and the second panel is spectral width (both in $\mathrm{m} / \mathrm{s}$ ).

the magnetic field, thus eliminating the development of an ambipolar electric field. Since we have not found a 3-D solution, we continue to rely on the 2-D analysis. We continue to do this because, models based upon the 2-D solution, so accurately describe many facets of the non-specular trail observations. In the future we hope to conduct 3-D simulations of meteor trails that will answer these questions, but conclude by suggesting that there are likely some trails that our 2-D model, and the results derived from it will not accurately describe.

Finally, we note that these estimates assume that radar reflections occur when and where a meteor trail becomes plasma unstable and turbulent. It is possible that some radar wavelengths, especially UHF and shorter are not particularly Bragg reflective from trail instability and turbulence that perhaps peaks near 3-5 m (Dyrud et al., 2002).

\subsection{ALTAIR observations}

To test whether measuring the altitudinal width of a nonspecular trail may be used to derive meteor velocity, we examined 6 examples of ALTAIR observations of head echos and non-specular trails. The ALTAIR radar is capable of measuring 3-D meteor velocities to within about $2 \mathrm{~km} / \mathrm{s}$ by examining the head echo range rate (Close et al., 2002). We have compared individual head echo range rates to the velocities obtained by measuring the width of the subsequent non-specular trail. These comparisons showed that the nonspecular trail technique resulted in velocities that matched to within $5 \mathrm{~km} / \mathrm{s}$ which is as a good as expected from such a technique. An example of this is presented in Fig. 1. The altitudinal width of this trail indicates the meteor that generated it was travelling at $60 \mathrm{~km} / \mathrm{s}$, while the head echo range rate indicates an actual meteor velocity of $63 \mathrm{~km} / \mathrm{s}$. While we only examined 6 examples, we find that when an unambiguous non-specular trail is present, the non-specular velocity technique produced no cases where the the two techniques differed by more than $5 \mathrm{~km} / \mathrm{s}$. This gives us a degree of confidence that this is a valuable technique. The next section demonstrates how hundreds of non-specular trails are capable of producing statistical results that also agree well with expectations.

\section{Piura radar observations and analysis}

To make a stronger case for the use of non-specular trails as a measure of meteor velocity, we present Piura radar observations of hundreds of non-specular meteor trails. Figure 5 plots SNR, Doppler shift and Doppler width of radar echoes 
Lower and Upper Bound Altitudes: 8-10-01, 4:00-6:00

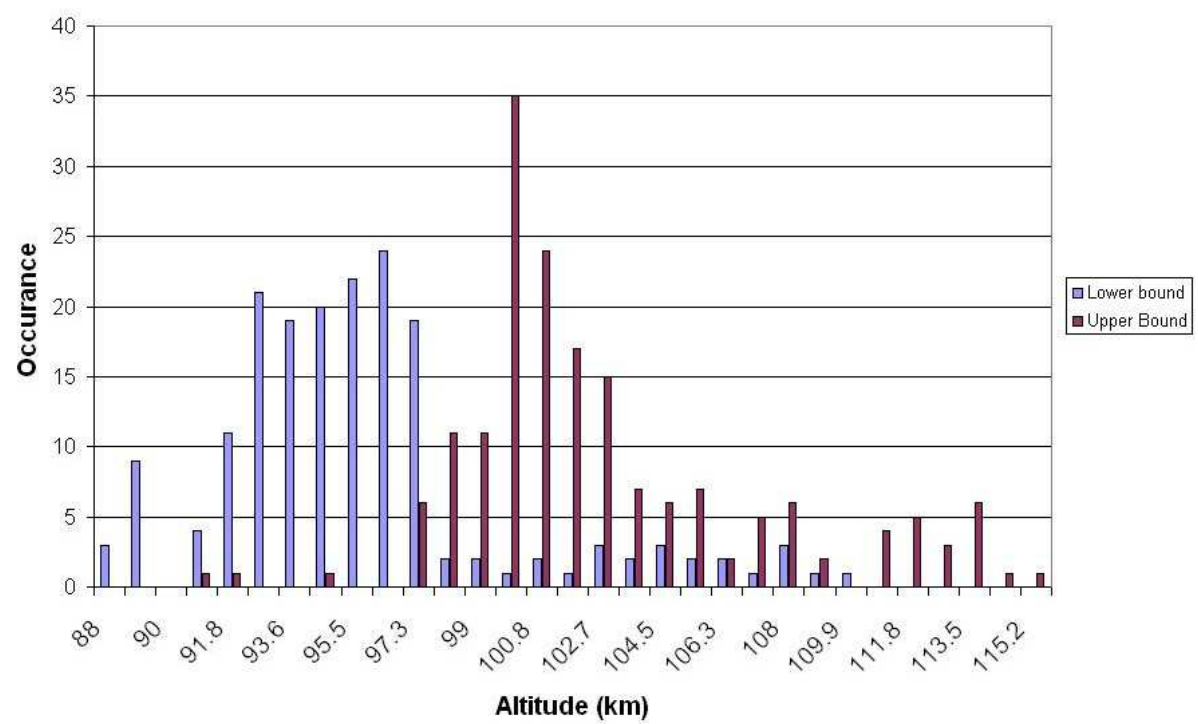

Fig. 6. Occurrence histogram of the upper and lower altitude extent of non-specular trails observed by the Piura radar. The plot shows that the most probable upper altitude for non-specular trails occurs at $102 \mathrm{~km}$, while the lower altitude is near $95 \mathrm{~km}$. These results are consistent with the altitude range observed using the ALTAIR radar data.

Piura Data: (4:00-6:00), 8-10-2001

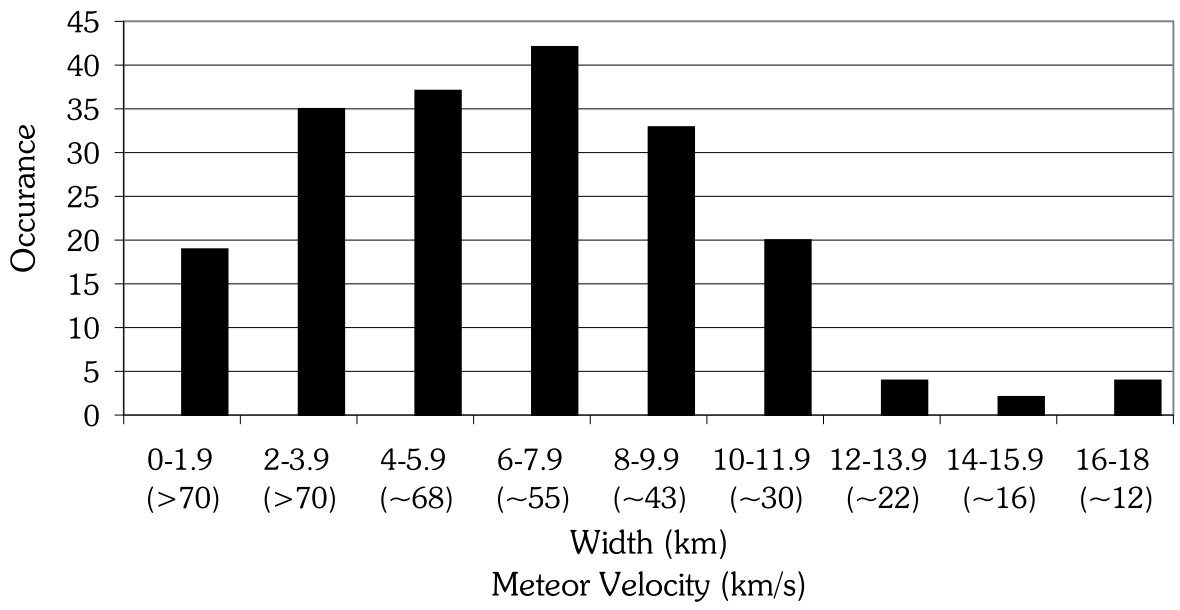

Fig. 7. Occurrence histogram of instability derived meteor velocity from $2 \mathrm{~h}$ of Radar data from 4:00-6:00 LT. The derived velocities show very reasonable results with few meteors below $30 \mathrm{~km} / \mathrm{s}$, which is expected for morning observations.

from the E-region obtained using the Piura radar system. The Piura radar is located in Peru at a geomagnetic dip angle of $7^{\circ}$ North, and operates at $50 \mathrm{MHz}$ using a phased array of COCO antennae. The data shown here was taken when the radar was pointed $14^{\circ}$ off-vertical to the North (i.e. within $1^{\circ}$ of perpendicular to B)(Woodman et al., 1999). These observations were made at a 5-s resolution and plotted at 1min averages. Such a scheme precludes the observation of head echos due to their very short lifetime and thus much lower average reflectivity. This figure plots two hours of data that shows vertical stripes near $100 \mathrm{~km}$ altitude nearly every minute. These isolated echos result from non-specular meteor trails, because head echos are too brief to be shown in this time averaged data. While not the topic of this paper, we point out here that the doppler shift of non-specular echos shown in the middle panel of Fig. 5 should correlate with the line of sight component of the neutral wind velocity. 
We have analyzed several features of the non-specular trails shown in these observations, such as the altitudinal width of each of the echos, and the upper and lower extent of the echos. We note here that we excluded electrojet echos based on their continuous signature and different Doppler shifts and widths. Isolated echos shorter than $1 \mathrm{~km}$ in range were also excluded from meteor counts, since such echos are either noise or specular meteor trail echos. Figure 6 plots the upper and lower altitudes of non-specular trails over $20 \mathrm{~h}$ of observations. The plot shows that the most probable upper altitude for non-specular trails occurs at $102 \mathrm{~km}$, while the lower altitude is near $95 \mathrm{~km}$. These altitudes are within $1 \mathrm{~km}$ of the ALTAIR observed altitudes of non-specular trails presented in Dyrud et al. (2002), and agree well with our theoretical predictions for the altitude range for meteor instability shown in Fig. 3.

Figure 7 shows an occurrence histogram for non-specular trail altitudinal width. The measure of width can then be converted to a meteor velocity using the theoretical relationship shown in Fig. 3. The converted velocity histogram peaks at about $55 \mathrm{~km} / \mathrm{s}$ with few observations below about $30 \mathrm{~km} / \mathrm{s}$. These results are consistent with observations of meteors in the morning and with head echo observations made at Arecibo (Mathews et al., 2001). While many head echo studies show a fast $(50 \mathrm{~km} / \mathrm{s})$ average velocity, scepticism exists because many researchers believe head echo observations are biased towards fast meteors. The non-specular trail technique, using data from $50 \mathrm{MHz}$ radar, while not suffering the same biases, should observe slow $(20 \mathrm{~km} / \mathrm{s})$ and fast meteors with nearly the same sensitivity. We base this prediction on the following theoretical work. Oppenheim et al. (2003b) showed that plasma columns with peak densities at least 10100 times the ionosphere are potentially unstable. Given this threshold Oppenheim et al. (2003a) and Dyrud et al. (2002) showed that meteor trail instability depends on trail density gradients and very weakly on peak densities. Therefore, non-specular trails resulting from plasma instability should be weakly sensitive to the meteor velocity that produced the trail. Finally, this work presents merely initial results of the usefulness of non-specular echos for deriving meteor parameters.

\section{Conclusions and summary}

The detailed observations provided by ALTAIR and the statistics from analyzing hundreds of non-specular trails from the Piura data set provide considerable evidence that non-specular trails result from the Farley-Buneman gradient drift instability driven turbulence described in Dyrud et al. (2002). This work shows that non-specular trails can be analyzed to provide information about the meteoroids that generated them. Specifically, we have shown that the altitudinal extent of a non-specular echo can be used to derive meteoroid velocity. This technique should prove useful for im- proving our estimates of the global flux of meteors at Earth, for the following reasons. Recent measurements of headechos show that the most probable head echo velocity is measured near $50 \mathrm{~km} / \mathrm{s}$ which contradicts previous estimates placing this velocity near $20 \mathrm{~km} / \mathrm{s}$ (Taylor, 1995). The findings from this study of non-specular trails also shows that the most probable meteor velocity lies near $50 \mathrm{~km} / \mathrm{s}$. However, controversy remains regarding head echo measurements. For example, Hunt et al. (2004) has shown that head echo detections are biased towards faster meteors, which is based on the correlation with ionization efficiency and head echo reflection. Regardless of the existence of these biases, nonspecular trail velocity measurements should not suffer the same issues, because very little ionization is theoretically required for a trail to become unstable and turbulent. Finally, the study presented here represents preliminary results and we continue to further validate this approach. To do this, one needs to systematically gather a larger set of combined head-echo and non-specular trail measurements, so that the correlation between these can be demonstrated over a wide range of conditions. Additionally, more sophisticated theoretical and/or numerical studies will complement these measurements. Nevertheless, our preliminary study indicates that this technique should prove useful in measuring and underemphasized population of small (approximately $\mu \mathrm{g}$ ) meteors at high velocities.

We conclude by suggesting some additional benefits of using non-specular trails to measure meteor velocity. When simultaneous head echos and non-specular trails are observed, the non-specular trail derived velocity may be applied to estimate 3-D meteoroid velocities in lieu of head echo interferometry. Additionally, cheaper, lower power radars pointed perpendicular to the magnetic field are capable of observing non-specular trails, but not head echos. These radars may now be used for the derivation of meteor velocity, and contribute to our global estimates of the distribution of meteor velocities.

Acknowledgements. The authors would like to thank Y. Dimont and $\mathrm{H}$. Petchek for their help and suggestions. This research was supported by NASA GSRP 500-092, NSF ATM 9986976, and the NSF CEDAR Post-Doc.

Edited by: D. Janches

\section{References}

Baggaley, W. J.: Single wavelength measurements fo the initial radii of radio meteor ionization columns, Bulletin of the Astronomical Institutes of Czechoslovakia, 32, 345-348, 1981.

Baggaley, W. J. and Webb, T. H.: The thermalization of meteoric ionization, Journal of Atmospheric and Terrestrial Physics, 39, 1399-1403, 1977.

Bronshten, V. A.: Physics of Meteoric Phenomena, D. Reidel Publ. Co., 1983. 
Brown, P., Simek, M., Jones, J., Arlt, R., Hocking, W. K., and Beech, M.: Observations of the 1996 Leonid meteor shower by radar, visual and video techniques, Monthly Notices to The Royal Astronomical Society, 300, 244-250, 1998.

Ceplecha, Z., Borovicka, J., Elford, W. G., Revelle, D. O., Hawkes, R. L., Porubcan, V., and Simek, M.: Meteor phenomena and bodies, Space Sci. Rev., 84, 327-471, 1998.

Chapin, E. and Kudeki, E.: Plasma-wave excitation on meteor trails in the equatorial electrojet, Geophys. Res. Lett., 21, 2433-2436, 1994.

Close, S., Hunt, S. M., Minardi, M. J., and McKeen, F. M.: Analysis of perseid meteor head echo data collected using the advanced research projects agency long-range tracking and instrumentation radar (altair), Radio Sci., 35, 1233-1240, 2000.

Close, S., Oppenheim, M., Hunt, S., and Dyrud, L.: Scattering characteristics of high-resolution meteor head echoes detected at multiple frequencies, J. Geophys. Res. (Space Physics), 107, 9-1, 2002.

Dyrud, L. P., Oppenheim, M. M., and vom Endt, A. F.: The anomalous diffusion of meteor trails, Geophys. Res. Lett., 28, 27752778, 2001.

Dyrud, L. P., Oppenheim, M. M., and vom Endt, A. F.: Interpretation of non-specular radar meteor trails, Geophys. Res. Lett., 29, 2002.

Erickson, P. J., Lind, F. D., Wendelken, S. M., and Faubert, M. A.: Meteor head echo observations using the millstone hill uhf incoherent scatter radar system, in Proceedings of METEOROIDS, 2001.

Heinselman, C.: Sondrestrom isr meteor measurements, in Proceedings of METEOROIDS, 2001.

Hunt, S., Close, S., Oppenheim, M. M., and Dyrud, L. P.: Two frequency meteor observations using the altair radar, in Proceedings of METEOROIDS, 2001.

Hunt, S., Close, S., Oppenheim, M. M., Brown, P., McKeen, F., and Minardi, M.: Determination of the meteoroid velocity distribution using high gain radar, Icarus, 168, 1, 34, 2004.
Janches, D., Mathews, J. D., Meisel, D. D., Getman, V. S., and Zhou, Q.-H.: Doppler Studies of Near-Antapex UHF Radar Micrometeors, Icarus, 143, 347-353, 2000a.

Janches, D., Mathews, J. D., Meisel, D. D., and Zhou, Q.-H.: Micrometeor Observations Using the Arecibo $430 \mathrm{MHz}$ Radar, Icarus, 145, 53-63, 2000b.

Jones, W.: Theoretical and observational determinations of the ionization coefficient of meteors, Monthly Notices to The Royal Astronomical Society, 288, 995-1003, 1997.

Lebedinets, V., Manochina, A., and Shushkova, V.: Interaction of the lower thermosphere with the solid component of the interplanetary medium, Planet. Space Sci., 21, 1317-1332, 1973.

Mathews, J. D., Meisel, D. D., Hunter, K. P., Getman, V. S., and Zhou, Q.: Very high resolution studies of micrometeors using the Arecibo $430 \mathrm{MHz}$ radar, Icarus, 126, 157-169, 1997.

Mathews, J. D., Janches, D., Meisel, D. D., and Zhou, Q.-H.: The micrometeoroid mass flux into the upper atmosphere: Arecibo results and a comparison with prior estimates, Geophys. Res. Lett., 28, 1929, 2001.

Oppenheim, M. M., vom Endt, A. F., and Dyrud, L. P.: Electrodynamics of meteor trail evolution in the equatorial E-region ionosphere, Geophys. Res. Lett., 27, 3173-3176, 2000.

Oppenheim, M. M., Dyrud, L. P., and Ray, L.: Plasma instabilities in meteor trails: Linear theory, J. Geophys. Res., 108, 7-1, 2003a.

Oppenheim, M. M., Dyrud, L. P., and vom Endt, A. F.: Plasma instabilities in meteor trails: 2-D simulation studies, J. Geophys. Res., 108, 8-1, 2003b.

Taylor, A. D.: The Harvard Radio Meteor Project velocity distribution reappraised, Icarus, 116, 154-158, 1995.

Woodman, R. F., Chau, J. L., Aquino, F., Rodriguez, R. R., and Flores, L. A.: Low-latitude field-aligned irregularities observed in the E-region with the Piura VHF radar: First results, Radio Sci., 34, 983-990, 1999.

Zhou, Q. H., Mathews, J. D., and Nakamura, T.: Implications of meteor observations by the mu radar, Geophys. Res. Lett., 28, 1399, 2001. 